\title{
The Genetic Variability of Wheat Can Ensure Safe Products for Celiac Disease Patients?
}

\author{
Miguel Ribeiro $^{1,2}$, Patrícia Poeta ${ }^{3,4}$, Gilberto Igrejas ${ }^{1,2, *}$ \\ ${ }^{1}$ Institute for Biotechnology and Bioengineering/Center of Genomics and Biotechnology, University of Trás-os-Montes and Alto \\ Douro, Vila Real, Portugal \\ ${ }^{2}$ Department of Genetics and Biotechnology, University of Trás-os-Montes and Alto Douro, Vila Real, Portugal \\ ${ }^{3}$ Center for Animal Science and Veterinary, Vila Real, Portugal \\ ${ }^{4}$ Department of Veterinary Science, University of Trás-os-Montes and Alto Douro, Vila Real, Portugal \\ *Corresponding author: gigrejas@utad.pt
}

Keywords: wheat, allergy, celiac disease, storage proteins, allelic variants

Wheat is a major source of protein in the human diet and presents an important genetic diversity that could be useful to look for cultivars with reduced allergenicity. It is the second largest crop annually produced and consumed ( $\sim 700$ million tonnes), and supplies about $20 \%$ of the world's calories. Wheat has the largest global production area ( 240 million ha) and the largest volume of a single crop traded internationally ( 140 million tonnes) [1]. Wheat endosperm proteins are subdivided into gliadins and glutenins. The glutenins confers elasticity to dough and consist of two types of proteins, the high-molecularweight glutenin subunits (HMW-GS) and the low molecular-weight glutenin subunits (LMW-GS), linked by disulfide bonds into large polymers with molecular weights that can exceed ten million Daltons. The gliadins are generally present as monomers and contribute with extensibility to wheat flour dough. These proteins are separated into alpha, beta, gamma, and omega gliadin subgroups, each with distinct primary protein sequences, and controlled by six main Gli loci located in the homeologous chromosomes of group 1 (Gli-1) and 6 (Gli-2) [2].

The absolute and relative amounts of these protein types vary considerably among cereals, as do their digestibility and immunogenicity. Cereal intolerance occurs at a prevalence of $1-3 \%$ of the population worldwide, but varies between countries. The causal proteins are the gliadins and the glutenins from wheat and related proteins from barley and rye, together commonly called 'gluten'. Gluten is one of the earliest protein fractions described as the "cohesive, visco-elastic proteinaceous material" that remains when wheat dough is washed to remove starch granules and water soluble constituents. Three pathologies are associated with gluten intake, respectively: i) food allergy that affects $0.2-0.5 \%$ of the population but has stronger clinical implications [3], ii) celiac disease (CD), which is an autoimmune disorder caused by the ingestion of gluten [4] affecting both children and adults throughout the world at various frequencies (from $0.1 \%$ to $>1.6 \%$ ), specifically about $1 \%$ of the Western world [5], and iii) gluten sensitivity, a pathology of intolerance to gluten that has been recently rediscovered [6], which excludes $\mathrm{CD}$ and wheat allergy, with an estimated prevalence of $6 \%$ for the USA population.
The pathologies associated with gluten have increased in recent years worldwide. Gluten intolerance (or celiac disease) is classified as an auto-immune disease and occurs in individuals with the human leucocyte antigen (HLA) DQ2 and/or DQ8 genotype [7]. Gliadins may be strong food allergens as these proteins may aggravate celiac disease [8]. The most CD-toxic epitopes are known from the gliadins (especially from the $\alpha$-gliadins). Diagnostic tools available are monoclonal antibodies, $\mathrm{T}$ cells, genomics and proteomics analysis, and reference material and volunteers are required. The ultimate diagnosis should be made upon histological evaluation of the small bowel mucosa. The epitopes from the $\alpha$-gliadins are considered to have by far the highest clinical relevance with regard to both the adaptive and innate response that lead to the development of celiac disease [9,10,11,12,13]. New diagnostic guidelines developed by ESPGHAN emphasize the crucial role of serological tests in the diagnostic process of symptomatic subjects, and of the detection of HLA DQ2/DQ8 alleles in defining a diagnosis in asymptomatic subjects belonging to at-risk groups. The serological diagnosis of $\mathrm{CD}$ is based on the detection of class IgA anti-tissue transglutaminase (antitTG) and anti-endomysial antibodies. In patients with IgA deficiency, anti-tTG or anti-deamidated gliadin peptide antibody assays of the IgG class are used. When anti-tTG antibody levels are very high, antibody specificity is absolute and CD can be diagnosed without performing a duodenum biopsy. Non-celiac gluten sensitivity is a gluten reaction in which both allergic and autoimmune mechanisms have been ruled out. Diagnostic criteria include the presence of symptoms similar to those of celiac or allergic patients; negative allergological tests and absence of anti-tTG and EMA antibodies; normal duodenal histology; evidence of disappearance of the symptoms with a gluten-free diet; relapse of the symptoms when gluten is reintroduced [14]. The exposure of the intestine to peptides derived from prolamins induces a $\mathrm{T}$ cell mediated inflammatory response, leading to destruction of villous structures. A sub-fraction of $\alpha$ gliadins, A-gliadins, is of special importance due to its severe CD allergenicity [15]. However, gliadins and glutenins are both active in celiac disease. Most research has been carried out on the repetitive domains of the $\alpha$ - 
and $\gamma$-type gliadins, with a number of epitopes being described. This focus may at least partly result from the availability of sequences of $\alpha$ - and $\gamma$-type gliadins and lack of sequences of $\omega$-gliadins. In fact, $\omega$-gliadins have also been shown to be active in CD $[16,17]$ and recent studies indicate that they contain immunodominant sequences which are also present in $\omega$-secalins and Chordeins [18].

Some short amino acid sequences have been reported as having powerful CD-immunogenic potential. All peptides found to be active in celiac disease contained the sequences Gln-Gln-Gln-Pro and Pro-Ser-Gln-Gln; inactive peptides contained no such sequences [8,15,19,20]. Moreover, a 33-mer peptide resistant to intestinal proteases and containing three of the most immunogenic epitopes was identified as one of the main stimulators of the inflammatory response to gluten [10,21].

There has been a substantial increase in the prevalence of celiac disease over the last 50 years and an increase in the rate of diagnosis in the last 10 years. Celiac disease can present with many symptoms, including typical gastrointestinal symptoms (e.g., diarrhea, steatorrhea, weight loss, bloating, flatulence, abdominal pain) and also non-gastrointestinal abnormalities (e.g., abnormal liver function tests, iron deficiency anemia, bone disease, skin disorders, and many other protean manifestations). Indeed, many individuals with celiac disease may have no symptoms at all [22]. To date, no pharmacological treatment is available to gluten-intolerant patients and the only effective treatment is a strictly life-long gluten-free diet, resulting sometimes in a social burden [23]. Dietary education should focus on identifying hidden sources of gluten, planning balanced meals, reading labels, food shopping, dining out, and dining during travel. Given the incomplete response of many patients to a GFD-free diet as well as the difficulty of adherence to the GFD over the long term, development of new effective therapies for symptom control and reversal of inflammation and organ damage are needed. The prevalence of celiac disease is increasing worldwide and many patients with celiac disease remain undiagnosed, highlighting the need for improved strategies in the future for the optimal detection of patients [22]. About 5\% of patients with celiac disease are refractory to a gluten-free diet. These patients should be referred to a gastroenterologist for reconsideration of the diagnosis or for aggressive treatment of refractory celiac disease, which may involve corticosteroids and immunomodulators [24]. In this sense, several promising strategies have been undertaken to reverse this scenario.

The gluten and gluten peptides can be hydrolyzed by prolylendopeptidases, allowing to produce celiac-safe food or even used as probiotic-driven enzymes, in which dietary gluten is hydrolyzed by coingested peptidases already in the stomach, thus preventing CD-specific immune reactions in the small intestine [25,26].

Microbial transglutaminase has also been used successfully to detoxify gluten by selective modification of glutamine residues present in CD-toxic epitopes [27]. One therapeutic strategy emerge by down-regulating the activity of zonulin - the active pump enabling gluten to enter the enterocytes [28]. Immune tolerizing therapies using a series of peptides which target the common genetic form of celiac disease (HLA-DQ2) are already available as the therapeutic vaccine Nexvax $2 \AA$.
On the other hand, the identification and/or breeding of wheat cultivars or close relatives naturally deficient in immunogenic prolamins are very appealing. The use of genetic engineering to down-regulate gene expression is now routinely used in many crops, including all the major cereals, and is therefore an attractive opportunity for reducing the immunotoxic components of gluten and, therefore the incidence of gluten-related allergies and intolerance in cereals. Most of the examples listed below of down-regulation of gluten proteins in wheat and other cereals are based on RNA interference (RNAi) technology [29].

Several current studies have shown that compared to current varieties, the percentage of old varieties and some landraces that have a lower CD-immunogenic potential is much greater [30,31,32], which in itself reveals the genetic potential of wheat for the discovery of new traits that enable the development of hypoallergenic varieties for celiac patients. The replacement of the unique technological properties of wheat gluten represents the major task of industry for providing high quality glutenfree foods, such as pasta, bread and baked products, in terms of structure, loss of starch during cooking, and optimal cooking time [33].

Nature offers a variety of cereals that are free of toxicity for gluten intolerant individuals, which include rice, corn, sorghum, millet, teff, and possibly some specific wheat varieties [29]. Sequencing studies of the wheat genome have produced, to date, draft sequences which should provide valuable tools for understanding and manipulating the gliadins in the future. The use of biodiversity, biotechnology and breeding ancient varieties may help to develop grains that have a low or zero content of immunotoxic sequences, but with reasonable baking quality. Using the latest technologies we expect to boost the development of hypoallergenic products with high technological and nutritional value that will be healthy and safe for Man.

\section{References}

[1] USDA, www.ers.usda.gov/data-products/wheat-data.aspx. 2013.

[2] Payne, P.I., Holt, L.M., Lawrence, G.J., Law, C.N., “The genetics of gliadin and glutenin, the major storage proteins of the wheat endosperm”, Plant Foods Hum. Nutr., 31. 229-241. 1982.

[3] Zuidmeer, L., Goldhahn, K., Rona, R.J., Gislason, D., Madsen, C., Summers, C., Sodergren, E., Dahlstrom, J., Lindner, T., Sigurdardottir, S.T., McBride, D., Keil, T., "The prevalence of plant food allergies: a systematic review”, J. Allergy Clin. Immunol., 121(5):1210-1218.e4. May 2008.

[4] Comino, I., Real, A., de Lorenzo, L., Cornell, H., López-Casado, M.A., Barro, F., Lorite, P., Torres, M.I., Cebolla, A., Sousa, C., "Diversity in oat potential immunogenicity: basis for the selection of oat varieties with no toxicity in coeliac disease”, Gut, 60(7). 915-922. Jul 2011.

[5] Abadie, V., Sollid, L.M., Barreiro, L.B., Jabri, B., "Integration of genetic and immunological insights into a model of celiac disease pathogenesis”, Annu. Rev. Immunol., 29. 493-525. 2011.

[6] Sapone, A., Lammers, K.M., Casolaro, V., Cammarota, M., Giuliano, M.T., De Rosa, M., Stefanile, R., Mazzarella, G., Tolone, C., Russo, M.I., "Divergence of gut permeability and mucosal immune gene expression in two gluten-associated conditions: celiac disease and gluten sensitivity”, BMC Med., 9. 23. Mar 2011.

[7] Gilissen, L.J.W.J., van der Meer, I.M., Smulders, M.J.M., "Reducing the incidence of allergy and intolerance to cereals", Journal of Cereal Science, 2014.

[8] Wieser, H., Relation between gliadin structure and coeliac toxicity. Acta Paediatr Suppl., 412. 3-9. May 1996. 
[9] Anderson, R.P., Degano, P., Godkin, A.J., Jewell, D.P., Hill, A.V.S., "In vivo antigen challenge in celiac disease identifies a single transglutaminase modified peptide as the dominant $\alpha$ gliadin T-cell epitope”, Nat Med, 6(3). 337-342. Mar 2000.

[10] Arentz-Hansen, H., Mcadam, S.N., Molberg, O., Kristiansen, C., Sollid, L.M., "Production of a panel of recombinant gliadins for the characterization of $\mathrm{T}$ cell reactivity in coeliac disease”, Gut, 46(1). 46-51. Jan 2000.

[11] Arentz-Hansen, H., Mcadam, S.N., Molberg, Ø., Fleckenstein, B., Lundin, K.E.A., Jorgensen, T.J.D., Jung, G., Roepstor V.P., Sollid, L.M., "Celiac lesion $\mathrm{T}$ cells recognize epitopes that cluster in regions of gliadins rich in proline residues”, Gastroenterology, 123(3). 803-809. Sep 2002

[12] Camarca, A., Anderson, R.P., Mamone, G., Fierro, O., Facchiano, A., Costantini, S., Zanzi, D., Sidney, J., Auricchio, S., Sette, A., Troncone, R., Gianfrani, C., "Intestinal T cell responses to gluten peptides are largely heterogeneous: implications for a peptidebased therapy in celiac disease", J Immunol., 182(7). 4158-4166. Apr 2009.

[13] Maiuri, L., Ciacci, C., Ricciardelli, I., Vacca, L., Raia, V., Auricchio, S., Picard, J., Osman, M., Quaratino, S., Londei, M., "Association between innate response to gliadin and activation of pathogenic T cells in coeliac disease”, Lancet, 362(9377). 30-37. Jul 2003.

[14] Tonutti, E, Bizzaro, N., "Diagnosis and classification of celiac disease and gluten sensitivity”, Autoimmun Rev., 2014 Jan 15. pii: S1568-9972(14)00055-X. 2014.

[15] De Ritis, G., Auricchio, G., Jones, H.H., Lew, E.J.L., Bernardin, J. E., Kasarda, D.D., "In vitro (organ culture) studies of the toxicity of specific A-gliadin peptides in coeliac disease”, Gastroenterology, 94(1). 41-49. Jan 1988.

[16] Camarca, A., Anderson, R.P., Mamone, G., Fierro, O., Faccchiano, A., Costantini, S., Zanzi, D., Sidney, J., Aurichchio, S., Sette, A., Troncone, R., Gianfrani, C., "Intestinal T cell responses to gluten peptides are largely heterogenous: implications for a peptide-based therapy in celiac disease”, J Immunol, 182(7). 4158-4166. Apr 2009.

[17] Ensari, A., Marsh, M.N., Moriarty, K.J., Moore, C.M., Fido, R.J., Tatham, A.S., "Studies in vivo of omega-gliadins in gluten sensitivity (coeliac sprue disease)”, Clin Sci (Lond), 95(4). 419424. Oct 1998.

[18] Tye-Din, J.A., Stewart, J.A., Dromey, J.A., Beissbarth, T., van Heel, D.A., Tatham, A., Henderson, K., Mannering, S.I., Gianfrani, C., Jewell, D.P., Hill, A.V.S., McCluskey, J., Rossjohn, J., Anderson, R.P., "Design of peptide-based immunotherapy and diagnostics for celiac disease based upon comprehensive, quantitative mapping of T-cell epitopes in gluten”, Sci Trans Med, 2(41). 41ra51. Jul 2010.

[19] Marsh, M.N., "Gluten, major histocompatibility complex, and the small intestine. A molecular and immunobiologic approach to the spectrum of gluten sensitivity "Celiac Sprue”,', Gastroenterology, 102(1). 330-354. Jan 1992.

[20] Maruyama, N., Ichise, K., Katsube, T., Kishimoto, T., Kawase, S., Matsumura, Y., Takeuchi, Y., Sawada, T., Utsumi, S., "Identification of major wheat allergens by means of the Escherichia coli expression system”, Eur. J. Biochem., 255(3). 739-745. Aug 1998.
[21] Shan, L., Qiao, S.W-, Arentz-Hansen, H., Molberg, Ø., Gray, G.M., Sollid, L.M., Khosla, C., "Identification and analysis of multivalent proteolytically resistant peptides from gluten: implications for Celiac Sprue”, J. Proteome Res., 4(5). 1732-1741. Sep-Oct 2005.

[22] Rubio-Tapia, A., Hill, I.D., Kelly, C.P., Calderwood, A.H., Murray, J.A., "ACG clinical guidelines: diagnosis and management of celiac disease”, Am J Gastroenterol., 108(5). 656676. May 2013.

[23] Kupper, C., "Dietary guidelines and implementation for celiac disease”, Gastroenterology, 128(4 Suppl 1). S121-127. Apr 2005.

[24] Pelkowski, T.D., Viera, A.J., "Celiac disease: diagnosis and management”, Am Fam Physician., 15, 89(2). 99-105. Jan 2014.

[25] Pyle, G.G., Paaso, B., Anderson, B.E., Allen, D.D., Marti, T., Li, Q., Siegel, M., Khosla, C., Gray, G.M.,"Effect of pretreatment of food gluten with prolyl endopeptidase on gluten-induced malabsorption in celiac sprue”, Clin Gastroenterol Hepatol., 3(7). 687-694. Jul 2005.

[26] Bethune, M.T., Khosla, C., "Oral enzyme therapy for celiac sprue”, Methods enzymol, 502. 241-271. 2012.

[27] Arentz-Hansen, H., Körner, R., Molberg, Ø., Quarsten, H., Vader, W., Kooy, Y.M., Lundin, K.E., Koning, F., Roepstorff, P., Sollid, L.M., McAdam, S.N." "The Intestinal T Cell Response to $\alpha$-Gliadin in Adult Celiac Disease Is Focused on a Single Deamidated Glutamine Targeted by Tissue Transglutaminase”, J Exp Med, 191(4). 603-612. Feb 2000.

[28] Paterson, B.M., Lammers, K.M., Arrieta, M.C., Fasano, A. Meddings, J.B., "The safety, tolerance, pharmacokinetic and pharmacodynamic effects of single doses of AT-1001 in coeliac disease subjects: a proof of concept study”. Aliment Pharmacol Ther, 26(5). 757-766. Sep 2007.

[29] Rosell, C.M., Barro, F., Sousa, C., Mena, M.C., "Cereals for developing gluten-free products and analytical tools for gluten detection”, Journal of Cereal Science.

[30] Molberg, Ø., Uhlen, A.K., Jensen, T., Flæte, N. S., Fleckenstein, B., Arentz-Hansen, H., Raki, M., Lundin, K.E.A., Sollid, L.M., "Mapping of gluten T-cell epitopes in the bread wheat ancestors: Implications for celiac disease”, Gastroenterology, 128(2). 393401. Feb 2005.

[31] Salentijn, E.M.J., Goryunova, S., Bas, N., Van Der Meer, I.M., Van Den Broeck, H.C., Bastien, T., Gilissen, L.J.W.J., Smulders, M.J.M., "Tetraploid and hexaploid wheat varieties reveal large differences in expression of alpha-gliadins from homoeologous Gli-2 loci”, BMC Genomics, 10:48. Jan 2009.

[32] Van Den Broeck, H.C., De Jong, H.C., Salentijn, E.M.J., Dekking, L., Bosch, D., Hamer R.J., Gilissen, L.J.W.J., Van Der Meer, I.M., Smulders, M.J.M., "Presence of celiac disease epitopes in modern and old hexaploid wheat varieties: wheat breeding may have contributed to increased prevalence of celiac disease”, Theor. Appl. Genet., 121(8). 1527-1539. Nov 2010.

[33] Lamacchia, C., Camarca, A., Picascia, S., Di Luccia, A., Gianfrani, C., "Cereal-Based Gluten-Free Food: How to Reconcile Nutritional and Technological Properties of Wheat Proteins with Safety for Celiac Disease Patients”, Nutrients, 6(2). 575-590. Jan 2014. 